\title{
Hot stuff
}

\section{Every scientist has an interest in their own citations, but regularly updated maps of citations across all of science are tracking what's hot and what's not.}

\section{Many physicists tend not to take} 'scientometrics', the discipline of measuring and analysing science research, seriously. Not everyone shares that view - an example of such scientometrics appeared recently in Science $^{1}$, in a paper exploring a model for citation dynamics across different journals and disciplines. And earlier this year, an interactive map ${ }^{2}$ of material published on the arXiv preprint server was released: colourcoded according to arXiv category, papers are represented by circles of a size proportional to their number of citations, clustered according to their references. Zooming in and out to explore the arXiv world is entertaining, but do these maps tell us anything useful?

Citation maps are, in fact, serious business. Thomson Reuters ScienceWatch, for instance, has been analysing science metrics since 1989, and so has the Japan National Institute of Science and Technology Policy (NISTEP) for the past ten years. Their methodology is based on clustering the top $1 \%$ of the most highly cited research papers each year. The resulting maps tell the story of active research areas in science and of the dynamics of different fields.

Hot topics come and go. For example, research into magnesium diboride - a conventional superconductor with an unconventionally high transition temperature - boomed in the early 2000 s and was a hot topic in NISTEP's science map for 2002; but by 2008 it had disappeared. Some areas of research are very dynamic, and a comparison between the 2002 and
2008 science maps shows clear trends - for instance, in condensed-matter physics there was a decisive change in hot topics; in this field, rapid increases in citations are common and research areas tend to develop independently. In contrast, for particle physics and cosmology the science map was almost unchanged, indicating no major shift in the pattern of research activity.

In a study ${ }^{3}$ by Chinese researchers performed in 2012, using the same methodology as the NISTEP reports, 132 active areas of research were identified across all of science. Of these, 31 were considered hot topics, and nine of them fell into physics or nanoscience categories: galaxy formation and evolution; metamaterials; slow light and silicon-based photonics; ultrafast lasers; ferroelectric, multiferroic and piezoelectric materials; cavity QED and solid-state quantum computation; fluorescence sensors and silicon carbide nanotubes; nanoparticles; and graphene. A more specific list ${ }^{4}$ of the hottest of the largest or most active topics in physics emerged in ScienceWatch's 2013 report: alkali-doped iron selenide; spinorbit coupled Bose-Einstein condensates; direct-detection experiments for dark matter; evidence of Majorana fermions; top-quark forward-backward asymmetry; quantum simulations using trapped ions; nodal gap structure in iron-based superconductors; holographic Fermi surfaces and entanglement entropy; interpretation of quantum discord; and topological insulators.
These lists indicate the level of activity in specific areas, and implicitly reflect the size of the communities involved (and hence should be interpreted with care). No doubt some of these topics will persist in the maps for years to come, others will disappear. But hot topics may also converge into new hot topics. Indeed, this 'movement' of research areas and their interactions are further interesting features revealed by the science maps - such as the confluence of ideas about Bose-Einstein condensation, superconductivity and superfluidity that is driving research on strongly interacting quantum many-body systems.

Science maps also show how activity is distributed in different countries and institutes, making their strengths and weaknesses plain. Hence, more funding bodies are using these data to assess the quality and impact of the research they pay for. With the ever-increasing number of publications - more than 26,000 articles were published in ISI-listed journals in physics in 2012 - the monitoring of trends in science will only become more important for researchers and funders alike. Scientometrics is most definitely on the map.

\footnotetext{
References

1. Wang, D., Song, C. \& Barabási, A-L. Science 342, 127-132 (2013). 2. http://paperscape.org

3. Pan, J., Zhang, X., Wang, X., Han, T. \& Wang, J. Mapping Science Structure 2012 (in Chinese) (Science Press, 2013).

4. King, C. \& Pendleton, D. A. Research Fronts 2013: 100 Top-Ranked Specialities in the Sciences and Social Sciences (Thomson Reuters, 2013); http://go.nature.com/59GqvF
}

\section{You know who}

\section{Remember, remember 23 November: it's 'The Day of the Doctor'.}

On 23 November 2013, the world marks 'The Day of the Doctor' - the Dr Who episode bearing that title and celebrating the fiftieth anniversary of the $\mathrm{BBC}$ television series will be broadcast simultaneously across the globe.

The time lord known only (so far) as 'The Doctor' hit British TV screens in 1963, the day after the assassination of US President John F. Kennedy. In 790 instalments since then he has battled daleks and cybermen, roaming through space and time in the TARDIS (from the outside, a 1960s London police box, although it's bigger on the inside) and 'regenerating' at intervals, such that the character has now been played by 12 actors from William Hartnell to Peter Capaldi, who takes over the role next month.

The popularity of the series is such that it is now regularly broadcast in 50 countries, and the anniversary episode will be carried by TV networks in 75 countries. More than 200 cinemas in the UK, 30 in Germany and 50 in Russia will screen the episode in $3 \mathrm{D}$, plus 100 cinemas in Australia and
New Zealand (although on 24 November). For BBC Worldwide, the commercial arm of the public broadcaster, $\mathrm{Dr}$ Who is one of its biggest brands: a down-turn in revenues for 2012-2013 is attributed, at least in part, to the production in that time-frame of fewer Dr Who adventures.

So let the celebrations begin - grab your sonic screwdriver and join us behind the sofa...

Published online: 17 November 2013 